\title{
Letter
}

\section{Enhancing effects of trichloroethylene and tetrachloroethylene on type I allergic responses in mice}

\author{
Makoto Seo ${ }^{1,2}$, Ryo Kobayashi', Tetsunori Okamura', Koji Ikeda', Masahiko Satoh ${ }^{1,3}$ \\ Naoki Inagaki ${ }^{4}$, Hiroichi Nagai ${ }^{4}$ and Hisamitsu Nagase ${ }^{1}$ \\ ${ }^{1}$ Laboratory of Hygienic Chemistry and Molecular Toxicology, Gifu Pharmaceutical University, \\ 1-25-4 Daigaku-nishi, Gifu 501-1196, Japan \\ ${ }^{2}$ Department of Biochemistry, Faculty of Medicine, Saitama Medical University, \\ 38 Morohongo, Moroyama, Iruma-gun, Saitama 350-0495, Japan \\ ${ }^{3}$ Laboratory of Pharmaceutical Health Sciences, School of Pharmacy, Aichi Gakuin University, \\ 1-100 Kusumoto-cho, Chikusa-ku, Nagoya, Aichi 464-8650, Japan \\ ${ }^{4}$ Laboratory of Pharmacology, Gifu Pharmaceutical University, 1-25-4 Daigaku-nishi, Gifu 501-1196, Japan
}

(Received December 17, 2011; Accepted January 12, 2012)

\begin{abstract}
Trichloroethylene (TCE) and tetrachloroethylene (perchloroethylene; PCE) are commonly identified as environmental contaminants of groundwater. Previously, we investigated the enhancing effects of TCE and PCE on antigen-induced histamine release and inflammatory mediator production in rat mast cells. In this study, to examine the potential effect of TCE and PCE on antigen-induced histamine release from mouse mast cells, mouse bone marrow-derived mast cells (BMMC) were sensitized with anti-dinitrophenol (DNP) monoclonal IgE antibody and then stimulated with DNP-BSA containing with TCE or PCE. Both TCE and PCE significantly enhanced antigen-induced histamine release from BMMC. Next we investigated the effects of TCE and PCE on the passive cutaneous anaphylaxis (PCA) reaction in vivo using ICR mice. TCE and PCE significantly enhanced the PCA reaction in a dose-dependent manner. In addition, we examined the enhancing effects of ingesting small amount of TCE and PCE in drinking water on antigen-stimulated allergic responses. After the ICR mice had ingested TCE or PCE in their drinking water for 2 or 4 weeks, we performed the PCA reaction. Both TCE and PCE ingestion enhanced the PCA reaction in a dose-dependent manner for 4 weeks. These results suggest that exposure to TCE and PCE leads to the augmentation of type I allergic responses in many species.
\end{abstract}

Key words: Trichloroethylene, Tetrachloroethylene, BMMC, Histamine release, PCA reaction

\section{INTRODUCTION}

In recent years, many epidemiological studies have shown that the incidences of allergic diseases such as asthma and pollinosis are increasing (Beasley et al., 2003; Frei and Gassner, 2008). Allergic diseases are caused by both genetic and environmental factors (Burney et al., 1990; Peat and $\mathrm{Li}, 1999)$. In particular, exposure to ambient environmental pollutants is known to be associated several allergic symptoms. For example, experimental evidence has shown that exposure to diesel exhaust particles (Inoue et al., 2007; Liu et al., 2008) and phthalate (Yanagisawa et al., 2008) increase allergic responses, and these pollutants have adjuvant activity.
Trichloroethylene (TCE) and tetrachloroethylene (perchloroethylene; PCE) are categorized as volatile organic compounds and are used as dry-cleaning and metal-degreasing agents in industrial operations. As a consequence of their widespread use, they are commonly identified as environmental contaminants of groundwater, soil, and indoor or outdoor air (Cooke, 1991; Westrick et al., 1984; Wu and Schaum, 2000). In humans, they are well known to disturb the central nervous system and induce changes in the parenchymal organs, especially the liver and kidneys (Lash et al., 2007; Wang et al., 2002). However, the immunotoxic effects of TCE and PCE are scarcely known. Previously, we reported the enhancing effects of TCE and PCE on antigen-induced 
allergic responses in vitro and in vivo using rats (Seo et al., 2008a, 2008b, 2008c). On the other hand, there have been no reports on the species differences in the enhancing effects of TCE and PCE on antigen-induced allergic responses.

In the present study, we investigated the effects of TCE and PCE on antigen-induced histamine release from mouse bone marrow-derived mast cells (BMMC). In in vivo studies, we investigated the effects of TCE and PCE on the passive cutaneous anaphylaxis (PCA) reaction using ICR mice. Furthermore, after the ICR mice had ingested low concentrations of TCE or PCE in their drinking water for 2 or 4 weeks, we performed the PCA reaction.

\section{MATERIALS AND METHODS}

\section{Reagents and animals}

TCE (purity 98\%) and PCE (purity 98\%) were purchased from Nacalai Tesque Co. Ltd. (Kyoto, Japan). The stock solutions (TCE; $30 \mathrm{~g} / 1$, PCE; $10 \mathrm{~g} / \mathrm{l}$ ) were prepared in dimethyl sulfoxide (DMSO). A mouse anti-dinitrophenol (DNP) monoclonal IgE antibody-residue was prepared by culturing EC1 cells, as reported previously (Sakurai et al., 1994). The EC1 culture supernatant was stored at $-80^{\circ} \mathrm{C}$ and used as a source of $\mathrm{IgE}$. The anti-DNP $\operatorname{IgE}$ antibody content of the preparation, as estimated by enzyme-linked immunosorbent assay, was $1.5 \mu \mathrm{g} / \mathrm{ml}$. DNP-conjugated bovine serum albumin (DNP-BSA) was prepared according to the previously reported method (Eisen et al., 1953). The number of DNP residues introduced was 13 per BSA molecule. Male ICR mice and male BALB/c mice were purchased from Japan SLC, Inc. (Shizuoka, Japan). They were housed four per cage and maintained on a 12-hr light: dark cycle. Water and a pelleted diet were supplied ad libitum. All experiments were performed according to the guidelines for the care and use of experimental animals produced by the Japanese Association for Laboratory Animal Science in 1987.

\section{Preparation and culture of BMMC}

Bone marrow cells were obtained by flushing the femurs of 6 week-old $\mathrm{BALB} / \mathrm{c}$ mice. The cells were cultured in RPMI-1640 medium (Gibco, Carlsbad, CA, USA) containing $20 \mathrm{ng} / \mathrm{ml}$ recombinant murine interleukin (IL)-3 (PeproTech, Rocky Hill, NJ, USA), 10\% heat-inactivated fetal bovine serum (FBS, Thermo Trace. Ltd., Melbourne, Australia), and $1 \%$ penicillin/streptomycin (Gibco) at $37^{\circ} \mathrm{C}$ in a humidified atmosphere containing $5 \% \mathrm{CO}_{2}$. Non-adherent cells were passaged every 5-6 days for 4 weeks. After
4 weeks, the recovered populations were composed of $>90 \%$ mast cells, as judged by an assessment of their morphology under Alcian Blue staining.

\section{Measurement of histamine release from BMMC}

The stock solutions of TCE and PCE were diluted with Tyrode solution (137 mM NaCl, $2.7 \mathrm{mM} \mathrm{KCl,} 1.6 \mathrm{mM}$ $\mathrm{CaCl}_{2}, 1 \mathrm{mM} \mathrm{MgCl}_{2}, 10 \mathrm{mM}$ HEPES, $0.05 \%$ gelatin, $0.1 \%$ glucose, $0.41 \mathrm{mM} \mathrm{NaH}_{2} \mathrm{PO}_{4}, \mathrm{pH} 7.4$ ) to a DMSO concentration of less than $0.1 \%$. In preliminary tests, we confirmed that this level of DMSO in Tyrode solution did not affect any of our experiments. The BMMC $\left(1 \times 10^{5}\right.$ cells $\left./ \mathrm{ml}\right)$ were sensitized by incubating them overnight at $37^{\circ} \mathrm{C}$ with $0.15 \mu \mathrm{g} / \mathrm{ml}$ anti-DNP IgE antibody in RPMI-1640 medium containing 2\% FBS. After washing the BMMC with Tyrode solution, they were pre-treated with various concentrations of TCE or PCE for $30 \mathrm{~min}$ at $37^{\circ} \mathrm{C}$. After treatment with TCE or PCE, the cells were stimulated with $10 \mu \mathrm{g} / \mathrm{ml}$ of DNP-BSA in Tyrode solution at $37^{\circ} \mathrm{C}$ for $30 \mathrm{~min}$ to induce antigen-stimulated histamine release. The supernatants of cell suspensions were then collected, and the histamine content of the supernatants was determined according to our previous method (Sato et al., 1998). Histamine release (\% of total) was calculated as the ratio of the fluorescence intensity of the sample to that of total histamine, and the percentage enhancement was calculated as the ratio of histamine release $(\%$ of total) in the presence of TCE or PCE to that observed in their absence.

\section{PCA reaction}

The stock solutions of TCE and PCE were diluted with phosphate buffered saline (PBS) to a DMSO concentration of less than $0.1 \%$. Various concentrations of TCE and PCE were intraperitoneally injected into the ICR mice ( 8 week-old). The mice were then intradermally injected with $40 \mu \mathrm{l}$ of anti-DNP IgE antibody diluted to 1:50 with saline into dorsal skin sites that had been shaved $24 \mathrm{hr}$ earlier. At $24 \mathrm{hr}$ after the passive sensitization, the mice were challenged intravenously with $200 \mu$ of antigenEvans blue solution (1 $\mathrm{mg}$ of DNP-BSA and $3 \mathrm{mg}$ of Evans blue were dissolved in $1 \mathrm{ml}$ of saline). The reaction was examined at $30 \mathrm{~min}$ after the challenge, and skin with blue spots was removed. PCA activity was determined by measurement of the concentration of Evans blue extracted from the cutaneous layer of the removed skin. The extraction of Evans blue was performed according to a previously described method (Katayama et al., 1978). Briefly, each pigment spot was placed in a test tube and incubated overnight at $37^{\circ} \mathrm{C}$ with $1 \mathrm{ml}$ of $1 \mathrm{~N} \mathrm{KOH}$, then $9 \mathrm{ml}$ of $0.6 \mathrm{~N} \mathrm{H}_{3} \mathrm{PO}_{4}$-acetone (5:13) were added, and the pigment 
TCE and PCE enhance type I allergic responses in mice

was extracted by mixing. After centrifugation, the amount of dye was determined colorimetrically (the absorbance at $620 \mathrm{~nm})$.

\section{TCE or PCE ingestion from drinking water}

The stock solutions of TCE and PCE were diluted with distilled water, and 0.03 and $3 \mathrm{mg} / 1 \mathrm{TCE}$ solutions and 0.01 and $1 \mathrm{mg} / 1 \mathrm{PCE}$ solutions were prepared and used as drinking water. The final concentration of DMSO in drinking water was less than $0.01 \%(\mathrm{v} / \mathrm{v})$. In preliminary tests, we confirmed that this level of DMSO in drinking water did not affect any of our experiments.

Eight-week-old ICR mice were treated with TCE or PCE in drinking water for 2 or 4 weeks. The vehicle group of mice was given distilled water. The water was changed every other day to ensure dose maintenance. Twenty-four hours before each treatment endpoint (2 or 4 weeks) of TCE or PCE ingestion, the mice were intradermally injected with $40 \mu \mathrm{l}$ of anti-DNP IgE antibody diluted to 1:50 with saline into dorsal skin sites. PCA reaction was performed as described above.

\section{Statistical analysis}

Data are shown as the mean \pm S.E. Statistical analy- ses were performed with StatView (SAS Institute, version 5.0). Fisher's protected least significant difference test or Schëffe's F test was applied, and a $P$-value $<0.05$ was considered significant.

\section{RESULTS}

\section{Enhancing effect of TCE and PCE on histamine release from antigen-stimulated $B M M C$}

To evaluate the enhancing effects of TCE and PCE on antigen-induced histamine release from BMMC, we examined histamine release from antigen-stimulated BMMC treated with TCE or PCE. Low concentrations of TCE and PCE significantly increased the amount of histamine released from the antigen-stimulated BMMC. Treatment with $0.1 \mathrm{mg} / 1 \mathrm{TCE}$ caused the biggest enhancement of histamine release from the antigen-stimulated BMMC (Fig. 1A, enhancement ratio compared with the control: 1.3). Moreover, treatment with PCE enhanced histamine release from the antigen-stimulated $\mathrm{BMMC}$ in a dosedependent manner (Fig. 1B, the peak enhancement ratio compared with the control: 1.7$)$.
(A)

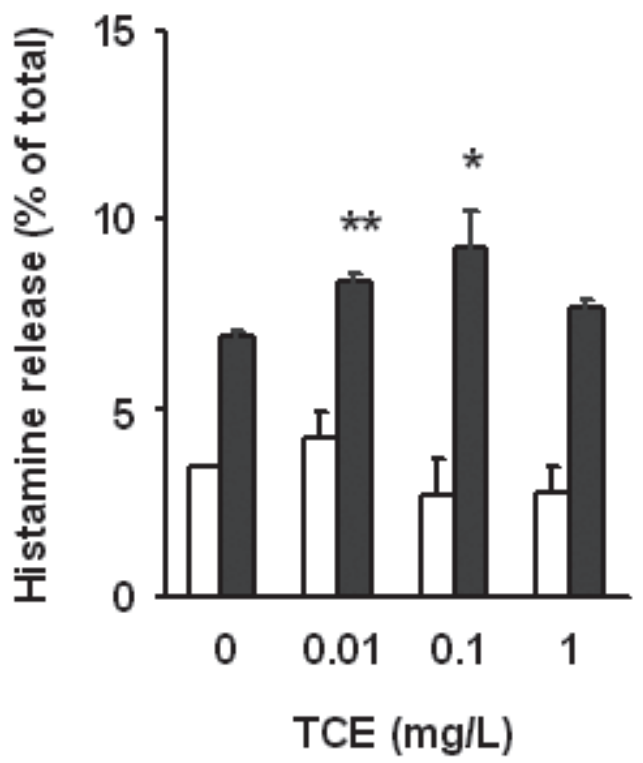

(B)

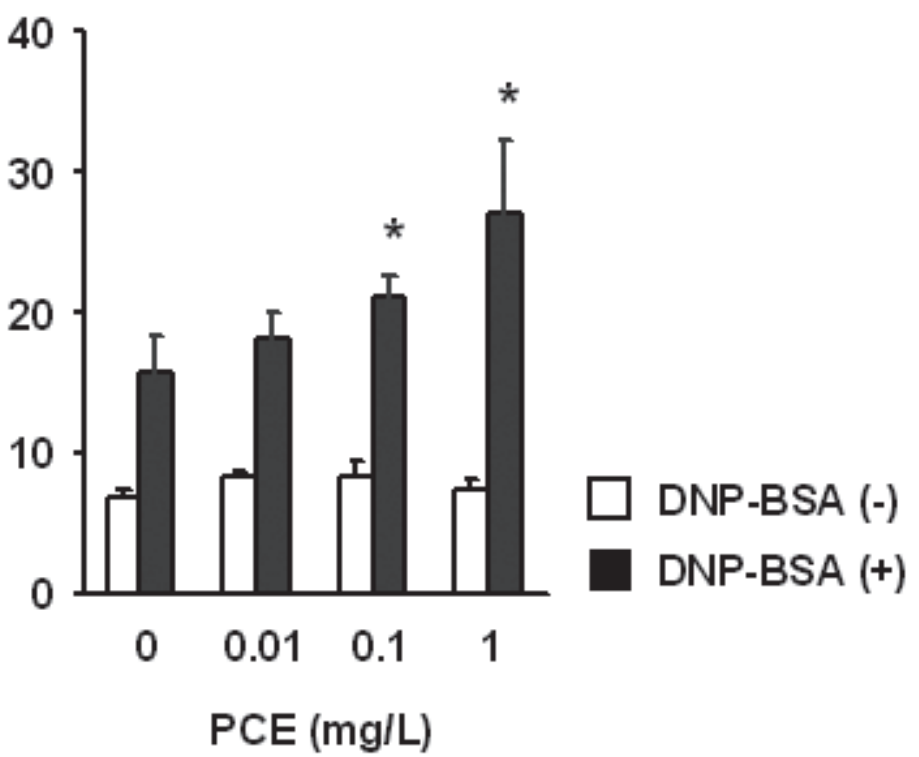

Fig. 1. Effects of TCE (A) and PCE (B) on antigen-induced histamine release from BMMC. After being sensitized with anti-DNP IgE, the cells were treated with TCE $(0.01-1 \mathrm{mg} / \mathrm{l})$ or PCE $(0.01-1 \mathrm{mg} / \mathrm{l})$ and then stimulated with DNP-BSA for $30 \mathrm{~min}$. The percentage histamine release was calculated as the ratio of the fluorescence intensity of the test sample to that of total histamine $(\mathrm{n}=3)$. Data are expressed as the mean \pm S.E. of 3 separate experiments $(* P<0.05, * * P<0.01)$. 


\section{Enhancing effects of TCE and PCE on the PCA reaction}

In our in vivo study, the effects of TCE and PCE on the PCA reaction were investigated. The results are shown in Fig. 2. Varying doses of TCE and PCE were intraperitoneally injected at $24 \mathrm{hr}$ before the antigen challenge. PCE and TCE significantly increased the PCA reaction in a dose-dependent manner, and the doses that had the strongest effects were $0.1 \mathrm{mg} / \mathrm{kg}$ body weight (b.w.) TCE (Fig. 2A, enhancement ratio: 2.0) and $1 \mathrm{mg} / \mathrm{kg}$ b.w. PCE (Fig. 2B, enhancement ratio: 2.6).

\section{TCE or PCE ingestion from drinking water for $\mathbf{2}$ or $\mathbf{4}$ weeks enhanced the PCA reaction}

The volumes of drinking water ingested by the TCE and PCE groups each day were not different from that ingested by the vehicle group (data not shown). In the TCE ingestion groups, each group of mice ingested a mean volume of drinking water of $6.0 \mathrm{ml} /$ day. This equates to a mean dose of $0.18 \mu \mathrm{g}$ TCE/day $(0.03 \mathrm{mg} / \mathrm{l}$ TCE treatment group) or $18.7 \mu \mathrm{g}$ TCE/day $(3 \mathrm{mg} / \mathrm{l} \mathrm{TCE}$ treatment group). In the PCE ingestion groups, each group ingested a mean volume of drinking water of $6.9 \mathrm{ml} /$ day. This equates to a mean dose of $0.07 \mu \mathrm{g} \mathrm{PCE} /$ day $(0.01 \mathrm{mg} / 1 \mathrm{PCE}$ treatment group) or $7.2 \mu \mathrm{g}$ PCE/day (1 mg/l PCE treatment group).

To evaluate the effects of TCE or PCE ingestion from drinking water on type I allergic reactions, we performed the PCA reaction. The results are shown in Figs. 3 and 4. The ingestion of 0.03 or $3 \mathrm{mg} / 1 \mathrm{TCE}$ in drinking water for 2 weeks significantly enhanced the PCA reaction in a dose-dependent manner (Fig. 3A, enhancement ratio: 1.5 at $0.03 \mathrm{mg} / 1 \mathrm{TCE}, 2.2$ at $3 \mathrm{mg} / 1 \mathrm{TCE}$ ), and a longer period (for 4 weeks) of TCE ingestion led to further enhancement (Fig. 3B, enhancement ratio: 2.3 at $0.03 \mathrm{mg} / \mathrm{l} \mathrm{TCE}$, 3.2 at $3 \mathrm{mg} / \mathrm{TCE}$ ). The ingestion of PCE in drinking water for 2 weeks did not enhance the PCA reaction compared with the vehicle treatment (Fig. 4A), whereas PCE ingestion for 4 weeks enhanced the PCA reaction in a dose-dependent manner (Fig. 4B, enhancement ratio: 2.1 at $0.01 \mathrm{mg} / 1 \mathrm{PCE}, 2.4$ at $1 \mathrm{mg} / 1 \mathrm{PCE})$.

\section{DISCUSSION}

In this study, we found that TCE and PCE markedly increased antigen-induced histamine release from mouse BMMC. In an in vivo study, a single intraperitoneal injection of TCE or PCE enhanced the PCA reaction, which showed a good correlation with the amount of histamine released from the antigen-stimulated BMMC. Furthermore, the ingestion of TCE or PCE from drinking water also enhanced the PCA reaction.

The lower concentrations of TCE and PCE used in our experiments represent the standard values of the Japanese standard for drinking water, which are similar to those outlined in the WHO drinking water guidelines (World
(A)

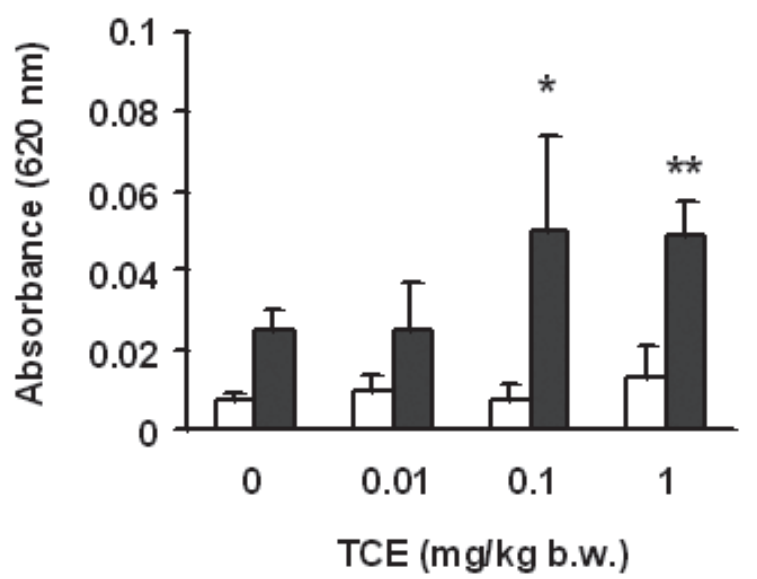

(B)

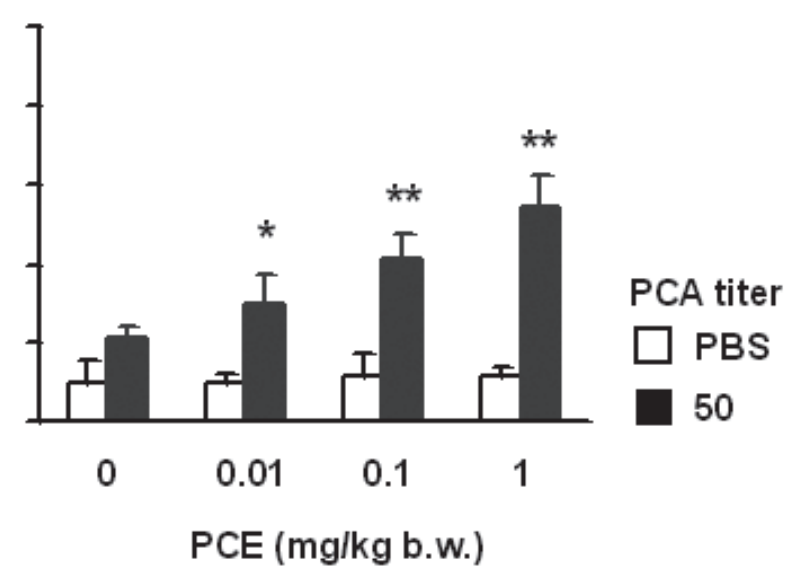

Fig. 2. Effect of TCE (A) or PCE (B) treatment on the PCA reaction. TCE (0.01-1 mg/kg body weight) or PCE (0.01-1 mg/kg body weight) was intraperitoneally injected at $24 \mathrm{hr}$ before antigen challenge. Two separate experiments were performed $(\mathrm{n}=4)$. Data are expressed as the mean \pm S.E. $\left({ }^{*} P<0.05,{ }^{* *} P<0.01\right)$. 
(A) 2 weeks

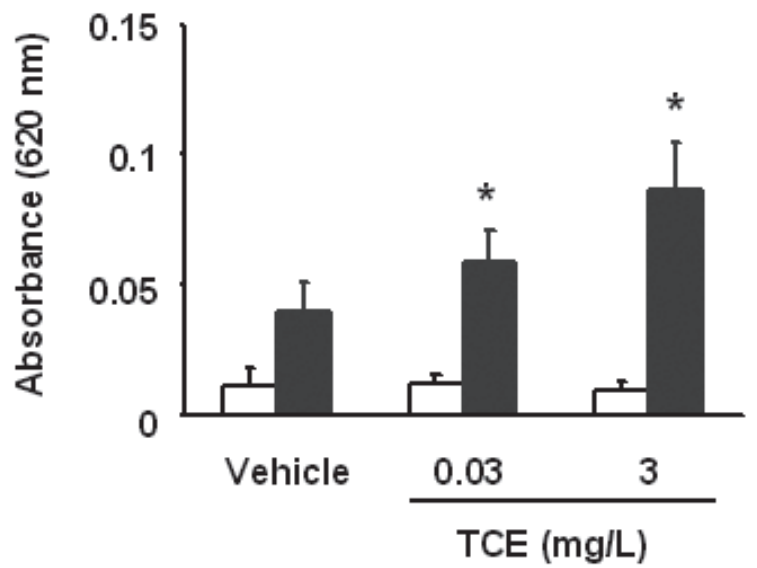

(B) 4 weeks

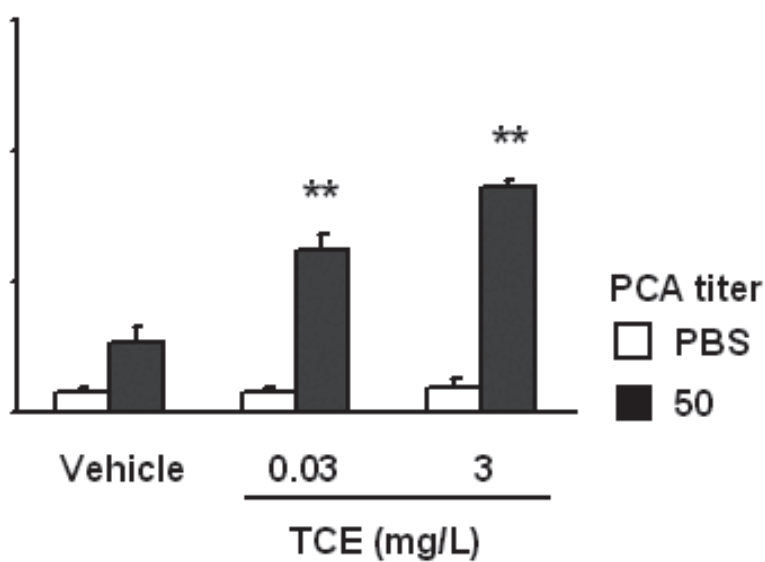

Fig. 3. Effects of ingesting TCE in drinking water for 2 or 4 weeks on the PCA reaction. Twenty-four hours before the end of treatment for 2 weeks (A) or 4 weeks (B), mouse anti-DNP IgE antibody diluted to 1:50 or PBS were intradermally injected. DNP-BSA-Evans blue solution was injected into the tail vein. Skin with blue spots was removed, and the supernatant of the Evans blue was measured. Data are expressed as the mean \pm S.E. $(* P<0.05, * * P<0.01)$.

(A) 2 weeks

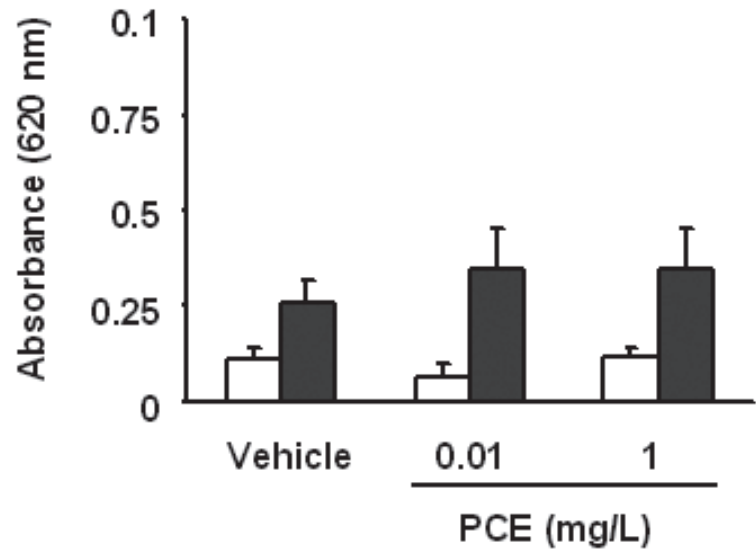

(B) 4 weeks

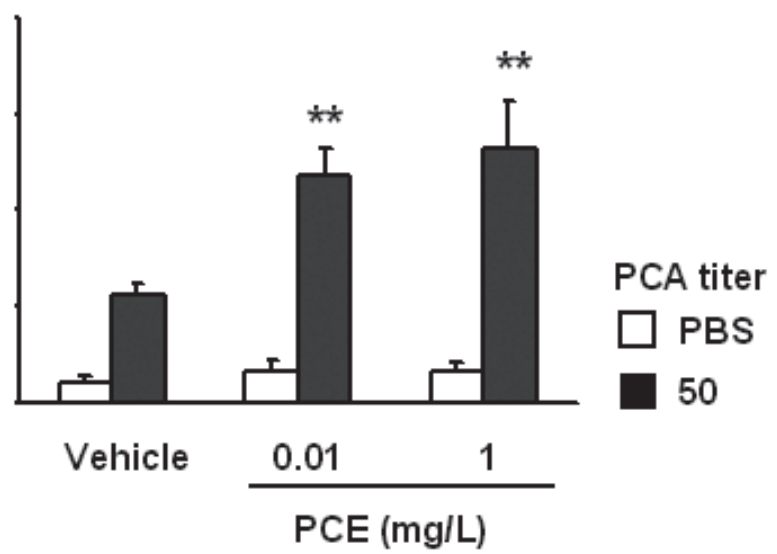

Fig. 4. Effects of ingesting PCE in drinking water for 2 or 4 weeks on the PCA reaction. Twenty-four hours before the end of treatment for 2 weeks (A) or 4 weeks (B), mouse anti-DNP IgE antibody diluted to 1:50 or PBS were intradermally injected. DNP-BSA-Evans blue solution was injected into the tail vein. Skin with blue spots was removed, and the supernatant of the Evans blue was measured. Data are expressed as the mean \pm S.E. $(* * P<0.01)$.

Health Organization, 1996). The other concentrations of TCE and PCE used in these experiments have sometimes been detected in groundwater from contaminated areas (Leschber et al., 1990; Wu and Schaum, 2000).
Our results show that TCE and PCE enhanced histamine release from antigen-stimulated BMMC. BMMC are mucosal mast cells that are distributed in the nasal, pulmonary, and gastrointestinal mucosa. In our previ- 
ous studies, the enhancement of antigen-induced histamine release by TCE and PCE was evaluated using connective mast cells, rat peritoneal mast cells (RPMC), and rat basophilic leukemia (RBL-2H3) cells. These different types of mast cells and basophils share many common features, such as they all express the high-affinity $\mathrm{IgE}$ receptor (FceRI), and activation of FceRI leads to histamine release and cytokine gene expression. From our present results, the enhancement ratio of histamine release from BMMC was slightly lower than those found for RPMC and RBL-2H3 cells. However, the pathogenesis of pollinosis is caused by the activation of mucosal mast cells; therefore, the enhancement of antigen-induced histamine release from BMMC by TCE and PCE is an important finding.

In our in vivo studies, it was shown that a single intraperitoneal injection of TCE or PCE markedly enhanced the PCA reaction in ICR mice compared with Wistar rats (Seo et al., 2008a). Furthermore, the ingestion of a low concentration of TCE or PCE from drinking water also markedly enhanced the PCA reaction in ICR mice compared with Wistar rats (Seo et al., 2008b, 2008c). In terms of metabolism, both TCE and PCE serve as substrates for cytochrome P450 (P450) and glutathione S-transferases, and the metabolic pathways of TCE and PCE are very similar (Lash et al., 2007). The kinetics of the formation of TCE and PCE metabolites are significantly different, and species differences in the rates of P450-dependent metabolism have been found, e.g., the rate of trichloroacetate (TCA) excretion is markedly slower in humans than in rats (Völkel et al., 1998). In our preliminary examinations, the main metabolites of TCE and PCE from the P450 pathway, TCA and chloral, did not enhance histamine release from antigen-stimulated BMMC, RPMC, or RBL-2H3 (data not shown). Therefore, we speculate that TCE and PCE themselves enhance antigen-induced allergic responses. When humans are transiently exposed to TCE or PCE, very little TCE or PCE accumulates in their tissues, as they are rapidly excreted into the urine and expelled during exhalation. However, chronic exposure to TCE or PCE may induce serious enhancement of allergic responses in humans. Further study is needed to confirm using human mast cells.

In conclusion, we demonstrated the enhancing effects of TCE and PCE on histamine release from antigen-stimulated $\mathrm{BMMC}$ and the PCA reaction in mice. These results suggest that the ingestion of TCE or PCE from drinking water enhances allergic responses in many species, even at low concentrations such as the values set for the Japanese standard for drinking water, and may accelerate the augmentation of allergic diseases.

\section{ACKNOWLEDGMENTS}

This research was supported in part by a Grant-in-Aid for Scientific Research from the Ministry of Education, Science, Sports, and Culture of Japan (no. 21590134).

\section{REFERENCES}

Beasley, R., Ellwood, P. and Asher, I. (2003): International patterns of the prevalence of pediatric asthma the ISAAC program. Pediatr. Clin. North Am., 50, 539-553.

Burney, P.G., Chinn, S. and Rona, R.J. (1990): Has the prevalence of asthma increased in children? Evidence from the national study of health and growth 1973-1986. Br. Med. J., 300, 13061310 .

Cooke, T.F. (1991): Indoor air pollutants. A literature review. Rev. Environ. Health, 9, 137-160.

Eisen, H.N., Belman, S. and Carsten, M.E. (1953): The reaction of 2,4-dinitrobenzenesulfonic acid with free amino groups of proteins. J. Am. Chem. Soc., 75, 4583-4585.

Frei, T. and Gassner, E. (2008): Trends in prevalence of allergic rhinitis and correlation with pollen counts in Switzerland. Int. J. Biometeorol., 52, 841-847.

Inoue, K., Takano, H., Hiyoshi, K., Ichinose, T., Sadakane, K., Yanagisawa, R., Tomura, S. and Kumagai Y. (2007): Naphthoquinone enhances antigen-related airway inflammation in mice. Eur. Respir. J., 29, 259-267.

Katayama, S., Shionoya, H. and Ohtake, S. (1978): A new method for extraction of extravasated dye in the skin and the influence of fasting stress on passive cutaneous anaphylaxis in guinea pigs and rats. Microbiol. Immunol., 22, 89-101.

Lash, L.H., Putt, D.A., Huang, P., Hueni, S.E. and Parker, J.C. (2007): Modulation of hepatic and renal metabolism and toxicity of trichloroethylene and perchloroethylene by alterations in status of cytochrome P450 and glutathione. Toxicology, 235, 11-26.

Leschber, R., Mergler-Vöelkl, R. and Nerger, M. (1990): Soil and ground water contamination by low boiling chlorinated hydrocarbons in Berlin. Int. J. Environ. Anal. Chem., 39, 159-164.

Liu, J., Ballaney, M., Al-alem, U., Quan, C., Jin, X., Perera, F., Chen, L.C. and Miller, R.L. (2008): Combined inhaled diesel exhaust particles and allergen exposure alter methylation of $\mathrm{T}$ helper genes and IgE production in vivo. Toxicol. Sci., 102, 76-81.

Peat, J.K. and Li, J. (1999): Reversing the trend: reducing the prevalence of asthma. J. Allergy Clin. Immunol., 103, 1-10.

Sakurai, T., Inagaki, N. and Nagai, H. (1994): The effect of antitumor necrosis factor (TNF)- $\alpha$ monoclonal antibody on allergic cutaneous late phase reaction in mice. Life Sci., 54, PL291PL295.

Sato, T., Taguchi, M., Nagase, H., Kito, H. and Niikawa, M. (1998): Augmentation of allergic reactions by several pesticides. Toxicology, 126, 41-53.

Seo, M., Ikeda, K., Okamura, T., Kida, K., Satoh, M., Inagaki, N., Nagai, H. and Nagase, H. (2008a): Enhancing effect of chlorinated organic solvents on histamine release and inflammatory mediator production. Toxicology, 243, 75-83.

Seo, M., Yamagiwa, T., Kobayashi, R., Ikeda, K., Satoh, M., Inagaki, N., Nagai, H. and Nagase, H. (2008b): A small amount of tetrachloroethylene ingestion from drinking water accelerates 
TCE and PCE enhance type I allergic responses in mice

antigen-stimulated allergic responses. Immunobiology, 213, 663669.

Seo, M., Yamagiwa, T., Kobayashi, R., Ikeda, K., Satoh, M., Inagaki, N., Nagai, H. and Nagase, H. (2008c): Augmentation of antigen-stimulated allergic responses by a small amount of trichloroethylene ingestion from drinking water. Regul. Toxicol. Pharmacol., 52, 140-146.

Völkel, W., Friedewald, M., Lederer, E., Pähler, A., Parker, J. and Dekant, W. (1998): Biotransformation of perchloroethene: Dosedependent excretion of trichloroacetic acid, dichloroacetic acid, and $\mathrm{N}$-acetyl-S-(trichlorovinyl)-L-cysteine in rats and humans after inhalation. Toxicol. Appl. Pharmacol., 153, 20-27.

Wang, F.I., Kuo, M.L., Shun, C.T., Ma, Y.C., Wang, J.D. and Ueng, T.H. (2002): Chronic toxicity of a mixture of chlorinated alkanes and alkenes in ICR mice. J. Toxicol. Environ. Health A, 65, 279291.

Westrick, J.J., Mello, J.W. and Thomas, R.F. (1984): The groundwater supply survey. J. Am. Water Works Assoc., 76, 52.

World Health Organization (WHO) (1996): Guidelines for drinkingwater quality, health criteria and other supporting information, second ed., Vol 2. World Health Organization, Geneva.

Wu, C. and Schaum, J. (2000): Exposure assessment of trichloroethylene. Environ. Health Perspect., 108, 359-363.

Yanagisawa, R., Takano, H., Inoue, K., Koike, E., Sadakane, K. and Ichinose, T. (2008): Effects of maternal exposure to di-(2-ethylhexyl) phthalate during fetal and/or neonatal periods on atopic dermatitis in male offspring. Environ. Health Perspect., 116, 1136-1141. 